\title{
The Mayinger Laboratory
}

Division of Nephrology \& Hypertension, Oregon Health \& Science University, Portland, OR, USA

\section{The People}

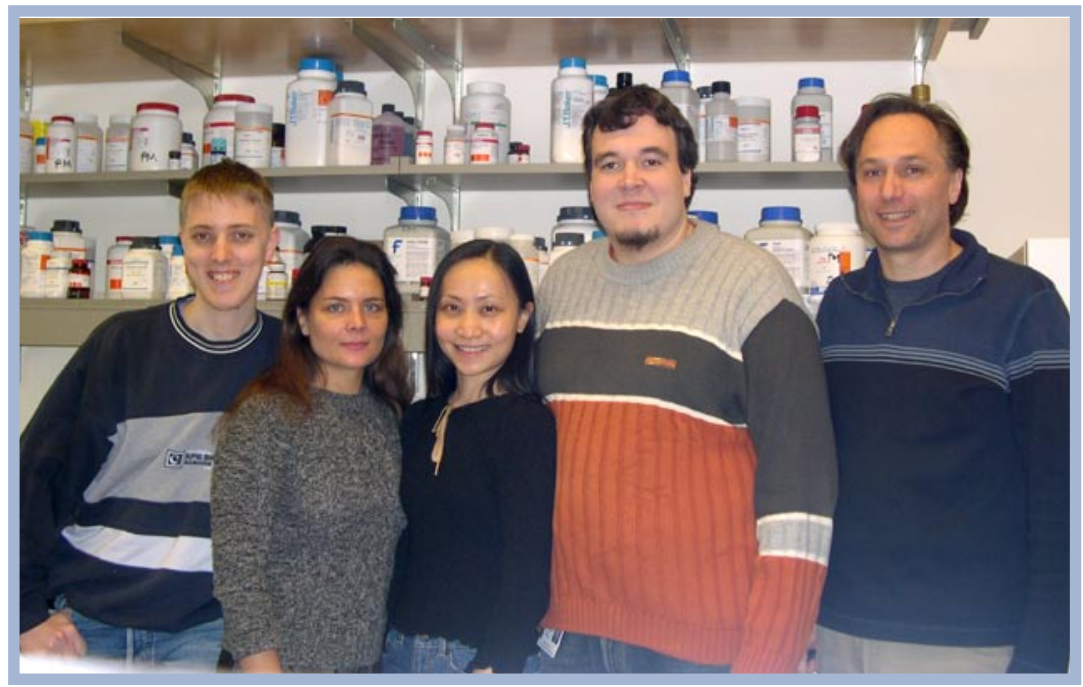

Left to right: Andreas Knödler (graduate student), Anastasia Blagoveshchenskaya (postdoc), Fei Ying Cheong (graduate student), Frank Faulhammer (graduate student), and Peter Mayinger (Assistant Professor of Medicine).

\section{The Research}

The Mayinger laboratory investigates lipid signaling pathways in health and disease. We study the cellular processes that are regulated by lipid signals, and we want to understand how the cellular levels of phosphorylated lipids are controlled by lipid kinases and phosphatases. Mutations in several lipid phosphatases are linked to severe human diseases, such as diabetes, kidney disease, and cancer, indicating that these enzymes play a particularly important role in regulating lipid signaling. Lipid phosphatases linked to membrane trafficking and organellar function are currently the main focus of our research. We examine how these enzymes contribute to the temporal and spatial control of membrane dynamics and analyze how malfunctions in these processes cause human disease. To this end, we use a combination of cell biological and biochemical tools in different model organisms. We have recently discovered that specific lipid phosphatases regulate endoplasmic reticulum (ER) and Golgi function in response to nutrients and cell growth rates. This mechanism is conserved in organisms from yeast to mammals. Our ongoing work is directed at elucidating how lipid signals coordinate the different intracellular processes that are important for cell growth and proliferation.

www.ohsu.edu/nephrology/faculty/mayinger/lab

\section{The Technique}

Phosphorylated derivatives of phosphatidylinositiol (phosphoinositides) regulate many cellular processes via downstream lipid-binding proteins. To analyze how phosphoinositide signaling networks operate, it is critical to identify the effector proteins that interact with phosphorylated lipids. In addition, the lipid-binding properties of such factors have to be characterized. Because deficiencies in lipid signaling pathways are related to severe human diseases, this analysis is also of considerable biomedical relevance. Most of the available methods for examining lipid-protein interactions are either relatively nonspecific or they require laborious preparations. We therefore developed a novel and simple protocol to study lipid-binding characteristics of proteins. This method uses a matrix of phosphoinositides reconstituted into biotinylated liposomes. The lipid composition that is used in liposome preparation can be varied easily and allows the reconstitution of a phospholipid environment similar to the one in intracellular membranes. Bound proteins can be rapidly recovered by affinity purification using streptavidin agarose. We currently use this method in our laboratory for identifying novel phosphoinositide-binding proteins and for characterizing how they interact with lipid membranes.

Analysis of phosphoinositide-binding proteins using liposomes as an affinity matrix, p. 858. 